\title{
Sistema de gestão do conhecimento para Rede Nacional de Bancos de Lei te Humano
}

\author{
Aknowledge management sys tem for the Brazilian \\ National Net work of Human Milk Banks
}

Paulo Ricardo da Silva Maia 1

Franz Reis Novak 1

João Aprígio Guerra de Almeida 1

Danielle Ap a recida da Silva 1

\footnotetext{
1 Banco de Leite Humano, Instituto Fernan des Figueira, Fiocruz.

Av. Rui Ba rbosa 716, 22250-020, Rio de Janei ro RJ. pmaia@fiocruz.br
}

\begin{abstract}
This article has as objective to demarcate concepts that can con tribute for enlargement of the theoretical chart for management of the knowledge in the REDEBLH. The structural bases for drawing the Kn owled ge Management Sys tem (KMS) a re also presen ted. It adopts a theoretical approach to present the foundations that in the literature maintain reflections about KMS. It's included still a short analysis of the setting of the knowled ge demarcated by the recent scientific output identified in the units from the net and in theirs headquarters. It expects that the questions discussed can make feasible the use of tools of share of the knowledge diminishing the deep social, economic, and cultural differences in the space of action from the REDEBLH.

Key words Knowledge management, Nets, Human banks of milk
\end{abstract}

Resumo Es te artigo teve como objetivos demarcar con cei tos que possam contri buir pa ra ampliação do quadro te ó ri co pa ra gestão do conhecimento na REDEBLH. São também apresentadas as ba ses estruturantes pa ra o desenho de um Sis tema de Gestão do Conhecimen to (SGC). Através de uma abordagem teó rica evidenciam-se os fundamen tos que na litera tu ra sustentam reflexões sobre SGC. O artigo inclui ainda uma breve análise do cen á rio do conhecimen to dem a rcado pela produção ci entífica recen te iden tificada nas unidades da rede e em sua sede. Espera-se que as questões discu tidas possam vi a bilizar o uso de ferramentas de compartilhamento do con hecimen to diminuindo as profundas diferenças cultu rais, e conômicas e sociais no espaço de atuação da REDEBLH.

Palavras-chave Gestão do conhecimento, Redes, Bancos de Leite Humano 


\section{Introdução}

A política pública de saúde, voltada para o incentivo à amamentação, tem, ao longo das últimas décadas, fortalecido a importância dos ban cos de lei te humano (BLH). Estas unidades configuram-se assim como local privilegi ado para as ações de incentivo ao aleitamen to materno no território nac i onal (Maia, 2004a).

O primei ro BLH do Brasil foi implantado em outubro de 1943 no então Instituto Nacional de Puericultura, atualmente Instituto Fernandes Figuei ra - IFF. O seu principal obj etivo era coletar e distribuir leite humano com vistas a atender os casos considerados especiais a exemplo da prematuridade, perturbações nutricionais e alergias a pro teínas heterólogas. Com esta mesma pers pectiva, en tre a década de $1940 \mathrm{e}$ o inicio dos anos 80, foram implantadas mais c in co unidades no país. Con tu do, foi com o des envolvimento do Programa Nacional de Incentivo ao Aleitamen to Matern o, sobretudo a partir de 1985, que os BLH passaram a assumir um novo papel no cenário da saúde pública brasileira, tran sforman do-se em el em en tos estratégi cos para as ações de promoção, pro teção e apoio à amamentação (Almeida, 2004).

Em 1998, foi criada, pelo Ministério da Sa úde, a Rede Nacional de Ba n cos de Lei te Humano (REDEBLH). Com sede na Fundação Oswaldo Cruz (Fiocruz), a rede, desde então, tem ampliado seu espaço de atuação tanto em função da permanente modernização de seu modelo de gestão, quanto pela geração de conhecimen to. Sua estrutura organizacional possui uma referência nacional, que está localizada na sede e é responsável pela normatização e proposição de políticas de atuação bem como pelas principais ações de des envo lvimen to cien tífico e tecnológi co. Complem entam esta estruturação as referências estaduais e as unidades locais (Maia, 2004a). Exis tecl a rorecon h ecim ento nacional dos avanços na saúde infantil proporci on ados pela REDEBLH. Com mais de 180 Bancos de Leite Humano implementados, a trajetória de sua con s trução indica dois fundam en tos que su stentam sua política de atuação. O primei ro é uma prática institucional com com promisso e responsabilidade social. O segun do diz res pei to ao exercício de solidariedade social, que ocorre por meio das doações voluntárias de leite humano ordenhado (LHO) que é distribuído, após processamento e rigoroso processo de controle de qualidade, para bebês prema tu ros de baixo peso intern ados em
Unidades de Tratamento In tensivo Neonatal (Maia, 2004a). Ben efícios econô micos também têm ocorrido na medida em que se estima dim i nuição na importação de lei te artificial para nutrir estes recém-nascidos impedidos, tem porariamente, de se alimentarem no seio materno.

A ampliação da REDEBLH foi acompanhada de nova con cepção de sua forma de atuação. De acordo com Al m eida (1998), há que se considerar, ainda, que estas alternativas deverão possibilitar um fluxo ágil e eficien te da informação, bem como possibilitar a universalização do acesso a ela.

Almeida (2001) identificou que o grande desafio con temporân eo para o de senvo lvimento da REDEBLH é a formação de competências para uma nova forma de pensar as questões relacionadas à amamen ta ção. Ne stesen ti do, a ponta três questões fundamentais: un iversalização do acesso dos profissionais aos novos saberes; o desenvolvim en to cien tífico e tecnológico; e a substituição do discurso ideológi co da amam entação por posições solidamen te ancoradas nos diferen tes campos do saber.

Tal indicativo demarcou, no campo da refl exão teórica, o início da sedimentação de um quadro con ceitual que objetiva, por um lado, su stentar novas políticas de atuação gerencial e de planejamento e, por outro, incorporar, no espaço acadêmico, novas possibilidades de teorização, particularmente na sede da REDEBLH.

Foi identificado também baixo grau de informatização e con ectividade eletrônica entre as unidades compon en tes. De acordo com Castells (2001), o desenvolvimento de redes só se tornou possível graças aos importan tes avanços tanto das tel ecomunicações quanto das tecnologias de integração de comp ut adores em rede. Assim, esta afirmação cria um paradoxo para a existência da REDEBLH.

Se o grau de con ectividade computacional é baixo, como é possívd e em que bases se dá a necessária integração para o funcionamen to em Rede? A hipótese cen tral do estu do é que o mecanismo de integração da REDEBLH tem como matéria-prima o conhecimento. Desta forma é necessário, para a estruturação de um sistema de gestão do con h eci m en to, com preender as dinâmicas dos processos de geração, distri buição e apropriação de ste conhecimento.

A partir do reconhecimento da importância estratégica do processo de elaboração concei tual, referi do às atividades de gestão tecnológica, que até então se con s ti túa de forma incipientee lateral, foi possível delimitar e el eger 
o campo da gestão do conhecimen to na REDEBLH como um novo e fundamental espaço pa ra construção do obj eto de estu do.

Para a formulação de alternativas que universalizem o acesso ao conhecimento, on de qu er que existam Bancos de Leite Humano em funcionamento e, da mesma forma, potencializar o ferra mental tecnológi cojá disponível na área, já foram desenvo lvidos estudos que oferecem uma com preensão mais ampliada do funcion amen to da REDEBLH (Maia, 2001; 2002).

Por outro lado, sabe-se que a dimensão continental do Brasil exige soluções que, em termos de saúde coletiva, possam compartilhar o conhecimen to acumulado nos gran des cen tros de formação e investi gação com os locais mais distantes (Maia, 2004b). Con tudo, s eg un doLópez (2001) é preciso enten der que as tecnologias da info rmação e da co municação não são igualitárias e se desenvolvem preferencialmente nos países mais desenvolvidos, dentro destes nas classes mais ricas e dentro destas, entre os próprios cidadãos, reproduzindo os padrões de desigualdade. Em síntese, a expressão destas desigualdades ocorre pela exclusão de grandes parcel a s populacionais da chamada sociedade do conhecimen to.

No Brasil, o Estado busca, por intermédio da promoção de políticas de inclusão social, exercer papel estratégico para que o des envolvimen to tecnológi co ben eficie, de forma eqüita tiva, as dimensões humana, ética e econômica (López, 2002).

O conhecimento deve tornar-se um dos principais fatores de superação de desigualdades, de agregação de valor, criação de em prego qualificadoe de prop a gação do bem-estar. Neste qu ad ro, ganha rel evância o de senvo lvi m en to de $\mathrm{n}$ ovos arc a bouços con cei tuais, m etodológicos e analíticos apropriados ao en ten dimen to de como os conhecimen tos produtivos são gerados, adquiri dos e difundido $s$, considera $n$ do as particularidades de países e regiões (Takahash, 2000).

Estas considerações, dianteda hipótese central do estudo, evidenciam a necessidade de ampliar a compreensão sobre as dinâmicas de geração e apropriação do conhecimento no â mbi to da REDEBLH. Tal opção se configura como etapa preliminar para o de s enho de um Si stema de Gestão doConh ecimen to (SGC).

Es te arti go, através de uma abord a gem teórica, apre senta fundamen tos que, na litera tura, sustentam reflexões sobre o assun to. Inclui-se ainda análise inicial do cenário do conhecimen to demarcado pela produção científica re- cente identificada nas Unidades da Rede e em sua Sede.

Vale ressaltar que o grande desafio desta abordagem é buscar um caminho que possibi lite, de forma inédita, aplicar con cepções teó ricas utilizadas em outros campos do saber à re alidade da REDEBLH.

\section{Referencial teórico}

As evidências con temporâneas revelam que está em curso um acel erado processo de transformação social. Nes te con tex to, estrutu ra-se uma n ova econ omia com pelo menos duas características bem fundadas: in formacional, porque a ativid ade dos agentes econômicos guarda estrita dependência com sua capacidade de gerar, processar e aplicar a informação baseada em conhecimentos; e global, porque, ta n to as atividades como seus componen tes estão organizados em dimensão mundial com forte tendência a arranjos or ganizacionais em rede.

O informacionalismo, na qualidade de novo modo de de s envo lvim en to, decorren te da ree struturação do modo capitalista de produção, tem como um de seus propulsores o avanço tecnológi co decorren te do acúmulo de conhecimento (Castells, 2001). Vale dizer que no informacionalismo a função da produção tecnológica se caracteriza pela con sta n te apropriação do conhecimento e da informação. A especificidade do modo inform aci i onal de de s envo lvimento está na ação do con hecimen to sobre o próprio conhecimen to, tornando-a principal fon te de produtividade. Decorre daí uma forte intera ção en tre cultu ra e forças produtivas que pode contribuir para a propagação de novos arranjos organizacionais como os cen trados no modelo de redes (Castells, 2001). As estruturas organizacionais centradas na integração e operação conjunta de vários atores, de processos produtivos de bens ou serviços, a exemplo das redes de inovação, têm sido consideradas as mais adequadas para promover a geração, aquisição e difusão do conhecimen to e inovações (Lastres, 2000). Os estu dos que privilegiam estes novos padrões de relacionamen to e gestão organizacional, com ênfase no conhecimen to e na inovação, apontam para o papel estratégico desempenhado pela ciência, pela tecnologia e pela inovação, na chamada econ omia de rede ou economia associacional (Lastres, 1999; 2000). As redes, neste senti do, ad qui rem extrema importância como ferramentas viabilizadoras do compartilha- 
men to do conhecimen to. Contudo, apesar do avanço da teleinformática, que hoje potencializa as possibilidades deste acesso, ainda não se verifica sua equalização. As profundas diferenças culturais, econômicas e sociais delimitam a capacidade de apropriação do conhecimen to disponibilizado.

Por outro lado, nos tempos atuais, não se pode negar que a informação, a inovação, a rapidez e a confiabilidade são con cei tos que delimitam os caminhos da coletivização do con hecimen to. O rompimen to de fron teiras até então intransponíveis, sedimentadas pelo modelo de desenvolvimento excludente, tem trazido como con seqüência maior acesso ao saber, mesmo que ainda limitado pelas condições sociais, políticas e econômicas já indicadas anteriormen te (Maia, 2004b).

É inegável que as chamadas tecnologias de mídia e a ampliação de seu alcance vêm crescendo a uma vel ocidade que não en con tra preceden tes na história recente. O volume de informações que circula diariamente no mundo vem aumentando vertiginosa e irreversivelmente. A título de ilu s tração, por volta de 1814, John Walter II, diretor do Times de Londres, instalou a primeira impressora a vapor capaz de imprimir mil jornais por hora. Apenas 10 anos mais tarde a imprensa da Grã-Bretanha atingiria a marca dos trinta milhões de exemplares (Virilio, 1996). Um período de qua renta anos foi necessário para que uma população de cinqü enta milhões de norte-americanos tivesse acesso ao rádio. No mesmo país, idên tico núm ero de pessoas já acessava seu Personal Computer $(P C)$, após quinze anos da introdução desta máquina. Mais recen temente, com o advento da Internet, em apenas quatro anos um número de usuários superi or a cinqüenta milhões de pessoas acessa a Web na quele país (Giden s, 2000).

O desenvolvimen to da ch a mada telecomunicação planetária tem produzido avanços na a propriação do conhecimento, contu do ainda são imensas as camadas sociais excluídas. Ne s te sentido ganha relevância o desafio de incluir, no processo de apropriação do con hecimento na REDEBLH, um número cada vez maior de atores sociais. Tal fato reforça a necessidade de se estabelecer matizes conceituais, que ofereçam opções teóricas para o des envolvimento da gestão do conhecimen to no âmbi to da REDEBLH.

A sociedade da informação (SI) tem sido objeto de estudo de um crescen te número de a utores (Castells, 2001; Lastres, 2000; Campos, 1997; Mansell, 1998). É nela que se estabel ecem as relações sociais e de de senvo lvimento tecnológico resultantes do avanço do conhecimen to humano.

No pres en te estu do, utilizou-se como referencial o con cei to de Socied ade da Informação de s envo lvido por López (2001) ou seja, um determinado nível de desenvolvimen to social, econômico e tecnológico caracterizado pela participação de diferentes agen tes (governo, empresas, pesquisadores, cen tros tecnológicos, organizações sociais e cidadãos) dispostos a gerar, difundir e usar a informação para produção do conhecimento econômico e socialmente útil (inovação) pa rafins do desenvolvimen to.

$\mathrm{Na}$ nova ordem econômica, o incremen to de produtividade, tanto nos processos como nos produtos, não depen de do aumen to quantitativo dos fatores de produção (capital, trabalho, recursos naturais) e sim da aplicação de conhecimentos e informação à gestão, produção e distribuição.

A sociedade da informação repre s enta uma profunda mudança na or ganização da sociedade e da economia. É um fen ô m eno global, com elevado potencial transformador das atividades sociais e econômicas. Assim é de se supor que ben efícios ou prejuízos para organizações e populações espalhadas nos diversos conti$n$ en tes podem re sultar do arranjo social decorren te do novo parad igma tecnológi co.

$\mathrm{O}$ acesso à informação, ao con hecimen to e, sobretudo, sua capacidade de aprender e inovar são os fatores da condição socioeconômica. Não basta uma base tecnológica e de infra-estrutura adequadas, também é necessário um conjun to de inovações nas estruturas produtivas e organizacionais, no sistema edu caci onal e nas instâncias reguladoras, norm a tivas e de governo (Takahash, 2000).

O processo de geração do conhecimen to também é afet ado por fatores ex ternos que influ enciam os rumos da evo lução científica. As mudanças verificadas nos processos produtivo $s$ e de trabalho, sobretu do como decorrência da chamada revo lução da microel etrônica e de todo o com plexo informacional computacional, con tribuem e, por vezes, condicionam as priorid ades da investigação.

Por sua vez, a sociedade globalizada, in tensamen te internacionalizada e interdepen den te, exige re adequações nas formas de fazer ciência. São alterados tanto os processos metodológicos, portanto internos à produção científica, 
como as situações e condições de trabalho (Minayo, 2002).

É assim que os avanços no campo da ciência ocorrem cada vez mais por meio da solução de problemas complexos apropriando-se não mais de uma, mas de várias disciplinas. Esta realidade con tribuiu para o surgimen to de sistemas de produção do conhecimento, socialmen te distribuído, caracterizados pelo trabalho em rede e cooperação diversificada, seja de indivíduos, grupos ou instituições (Pelegrini, 2000).

A configuração de redes de conhecimento tem como pressuposto, por um lado, a identificação do con hecim en to acumu l ado e disponível e, por outro, uma demanda para sua aplicação. Além de s tes condicionantes, é neces sá rio um interesse comum que possa proporcionar vantagens com peti tivas para os atores (Merin o, 2002).

Examinando a produção do con hecimento, do pon to de vista intern o, podem ser verificados novos padrões e tendências. A ampliação das possibilidades trazidas pelo modelo da big science dissolve, na prática, a antiga dico tomia en tre ciência básica e aplicada (Minayo, 2002).

\section{Do con hecimento à gestão do conhecimento}

O conhecimen to tem sido preocupação histórica da epistemologia e existe con s enso de que é um termo de difícil definição. Ao mesmo tempo é secular o esforço de filósofos para compreensão dos processos de geração e apropriação do conhecimento. Alguns dos principais pen sadores como Sócrates, Platão e Aristóteles ofereceram con tri buições ao tema que até hoje influenciam o pensamento moderno (Kane, 2003; Ma rcon des, 2002).

No sistema aristotélico, o conhecimento pode ser en ten dido como saber teóri co que se divide em ciência geral e ciência natural; como saber prático (práxis), que inclui a ética e a política e o saber produtivo (poiesis), que seria a base do estudo de estética. Com estes fundamentos, o filósofo des envo lveu uma con cep ção sistemática de saber com marcante influência na An tiguid ade (Ma rcon des, 2002). Con tu do, é o projeto filosófico de Descartes que exerce mais forte influência nas formulações con ceituais acerca do conhecimen to que identificamos em nossa época. O con fli to en tre dois modelos de ciência, o antigo e o modern o, vivenciado pelo filósofo no inicio do século 17, e as incertezas daí resultantes o estimularam a assumir a missão de legitimar a ciência a partir do entendimen to de que o homem pode conhecer o real de modo verd adeiro e definitivo. O proj eto de Descartes pretendia fundamentar a possibilidade do conhecimen to científico da nova Ciência encontrando uma verdade inquestionável e refutan do o ceticismo (Ma rcondes, 2002). Segundo Allix (2003), este pensamen to exerceu forte influência nos conceitos acerca do conhecimento adotados por importantes teó ricos con temporân eos que trabalham com gerência do con hecimen to (Nonaka, 1994; Nonaka \& Takeuchi, 1995; Nonaka et al., 2001).

$\mathrm{Na}$ década de 1960, o estudo de Michael Polanyi (1966) representou, do pon to de vista epistemológi co, uma das fundamentais con tribuições à discussão sobre natu reza do con h ecimento. Mais recentemente, o arti go de Moore \& Bolinches (2001) sistematiza alguns dos principais esforços de con ceituação. Os autore s assinalam que não existe uso ex a to para a palavra conhecimen to, portan to pode-se construir muitas formas para sua aplicação. Em seu estudo apresentam um en foque que predomina no pensamento contemporâneo sobre o tema e sugerem um esquema compreen sivo para o conceito de conhecimen to centrado em duas dimensões: a tácita e aexplícita.

A dimensão tácita do conhecimento diz respeito tanto ao que sabemos, porém não exteriorizamos de maneira formal, como também qua n to àquilo que sabemos, porém ainda não temos consciência. Ou ainda pode ser en ten dido como aqu ele conhecimento ineren te ao ser humano, que não está estruturado de forma perceptível a exemplo das experiências dos indivíduos, suas habilidades, s eu know-how, suas práticas, s eus valores (Valen tim et al., 2003). Já o conhecimento explícito é formal, estruturado, ex presso em símbolos e em processos e procedimen tos que podem ser codificados e decod ificados por aqueles que conhecem as leis, regras e métodos de uma disciplina científica ou de um campo profissional. A tecnologia é talvez o melhor exemplo deste conhecimento.

Ne s te sen tido pode-se afirmar que o con hecimen to existe em forma tácita na mente das pessoas, da qual em erge na forma explícita em resposta a problemas e desafios de natureza pró pria ou ex terna (Nahapiet\& Ghoshal, 1998). A origem dos problemas pode ser uma mera curiosidade intelectual ou uma necessidade que su rgecomo conseqüência da relação de uma organização com seu en torno. 
A gestão do conhecimen to atua essencialmen te nos flu xos informais de informação e no conhecimen to tácito (Valen tim et al. , 2003). Os estudos sobre possíveis combinações de criação e transmissão do conhecimen to, considerando as dimensões tácita e explícita, foram realizados nos anos 90 principalmen te por Nonaka y Takeu chi (1995). O modelo, por eles des envolvido, de base epistemológica, leva em consideração a existência de qua tro processos básicos geradores de conhecimento: socialização; ex temalização; com binação e internalização (SECI) como descritona figura 1.

O utras investigações privilegiam a dimensão definida como ontológica. Estes estudos levam em consideração as entidades que são ca- pazes de produzir conhecimen to, e con si deram quatro níveis possíveis: o individual, ou seja aqu ele conhecimento cri ado pelo próprio indivíduo; o grupal, derivado das interações entre pessoas; os níveis organizacionais, que integram todos os conhecimen tos dos setores da or ganização e o interorganizativo, que resulta da interação da or ganização com os agen tes em seu en torno (Nahapiet \& Ghoshal, 1998; Nelson, 1982; S pen der, 1996). O con cei to de on tologia tem sido utilizado nos estu dos envo lvendo inteligência artificial e repre sentação do conhecimen to. Ontologia neste caso é em pregada no sentido de formular um exa us tivo e rigoroso esquema conceitual de um domínio dado, com vistas a facilitar a comunicação e o com-

\section{Figura 1}

Processos de conversão do conhecimento.
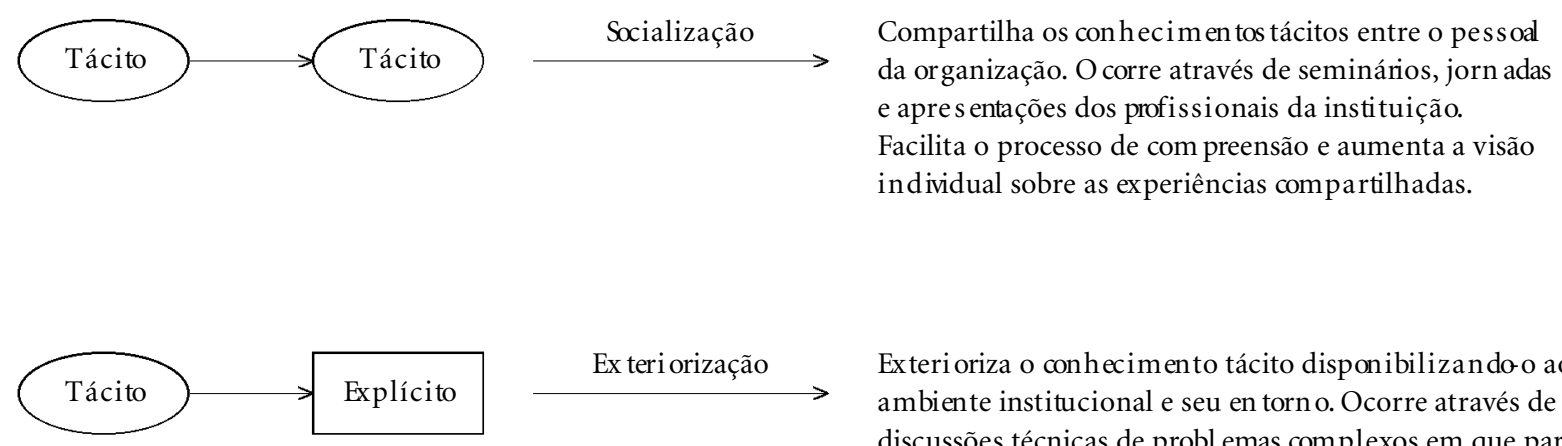

Exteri oriza o conhecimento tácito disponibilizando-o ao ambiente institucional e seu en torn o. Ocorre através de discussões técnicas de probl emas complexos em que participam profissionais do Ins ti tuto e de outras empresas. Propicia abusca de soluções técnicas aos probl emas dos dientes e a transferência de resultados e tecnologia.

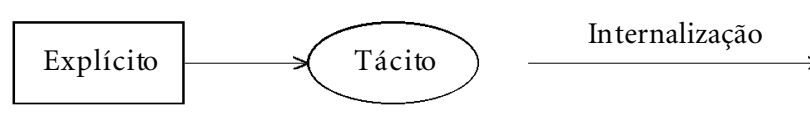

Proporci iona a refl exão a partir de experiências realizadas.

O corre através do trabalho em redes on de os profissionais podem explorar na prática as soluções des envolvidas para determinados probl emas. Consolida os processos de apren dizagem indivi dual e coletiva da or ganização.
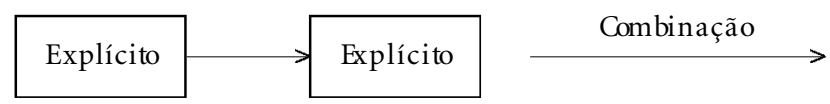

In tegra as soluções técnicas já desenvo lvidas e con h ecidas pela or ganização para enfrentar probl emas de maior complexidade. O corre através da con strução de protótipos, modelos e outras formas. Facilita a sistematização, registro e codificação das principais linhas de produtos e serviços da organização. 
partilhamento do conhecimento e da informação entre diferen tes sistemas. A consideração da dimensão ontológica permite determinar que entidades são capazes de criar conhecim ento e aqu elas que são capazes de apren der. Portanto, o conceito de ontologia aqui adotado guarda diferenças com o significado filosófico do term o.

A proposta metodológica de López (2002) utiliza um modelo integrado para o entendimento da criação e transformação do conh ecimen to. Traz novas e fundamentais con tribuições para o desenvolvimen to concei tual do tema. A partir de uma análise crítica dos modelos explicativos de senvo lvi dos na última década, em especial o de Sa n chez (2001), são estabelecidas as bases conceituais para o modelo EO-SECI (Epistemological \& Ontological SECI), integrador das diferen tes corren tes de pensam en to. Busca a articulação do modelo de base epistemológica com a proposição de sustentação ontológica. Traz para o mesmo campo de análise a natu reza do conhecimento e os níveis ontológi cos que compõem os distintos organismos que geram conhecimento. Descreve quatro níveis nos quais é possível identificar o desenvolvimen to de processos de criação do con hecimento: o individual, o grupal, o organizacional e o interorga niza tivo. O modelo objetiva analisar os processos que se produzem no interi or de cada um dos níveis e suas relações.

O nível mais baixo dos fatos conhecidos são os dados e estes não possuem um significado intrínseco. Quando os dados são processados a través de sua ordenação, grupamen to, análise e interpretação se convertem em informação. Por outro lado, quan do a informação é utilizada e colocada num con texto ou marco de referência de uma pessoa tra $\mathrm{n}$ forma-se em conhecimento. O conhecimento seria assim, a combinação de informação, con tex to e ex periência (Zorrilla, 1997).

Do ponto de vista conceitual é necessário ainda de stacar as diferenças en tre gestão do conhecimen to e gestão da informação como a pontadas em Salazar (2001).

Para con secução dos obj etivos deste artigo é também importante en tender o concei to de Sistema de Gestão do Conhecimen to (SGC). A literatu ra especializada destaca dois enfoques que podem balizar as definições de SGC. No viés organizacional a ênfase está na com preensão e sistem a tização dos processos medianteos quais as pessoas adquirem e geram conhecimen to.
A outra linha de pensadores, que adota o enfoque econômico, centra esforços nos processos de gestão do conhecimento potencialmen te geradores de exceden tes econômicos. Pa ra estes, o conhecimen toé vis to em seu processo de criação de valor patrimonial e vantagens competitivas. Ambos os en foques são, na realidade, complementares (Zorrilla, 1997; Salazar, 2001).

A definição con ceitual do Sistema de Gestão do Conhecimen to para a Rede Nacional de Bancos de Lei te Humano foi descrita como: espaço criado pela Rede a partir de uma visão integral da problemática da saúde pública em sua á rea de competência, com a finalidade de potencializar o capital intelectual da Rede para implantar os processos e procedimentos que facilitem o acesso às diversas formas de conhecimento necessárias ao melhor desem penho de suas Unidades (Maia, 2004b).

As principais funções de um SGC podem s er repres en tadas como indicado na figura 2 .

Cadasubsistema (vigilância, c riação e transferência) po s sui funções específicas que se rel acionam num movimen to de perman ente interatividade no interior do SGC. Cada el em en to do SGC, num ambiente de rede de inovação, pode desempenhar uma ou mais funções como s erá mostrado mais adian te(Maia, 2004b).

\section{Metodologia}

Este estudo foi conduzido em três etapas: 1a definição do modelo teórico de Sistema de Gestão do Conhecimento; 2 a - diagnósti co situacional e $3 \underline{a}$ - realização de uma simulação para avaliar a aplicabilidade do modelo propos to.

O modelo teóri co selecionado foi o desenvolvi do por Moore \& Bolin ches (2001), que es tabel ece qua tro com pon en tes estrutu ra $n$ tes para um SGC assim descritos em Maia (2004b):

- Acoleção: deve con ter as bases de dados, as imagens, os documentos, vídeos, a presentações, experiências práticas, as informações e os conhecimentosexplícitos requ eridos pelo neg ó ci o; - A infra-estrutura de comunicação: con stituída pela rede de informática necessária para estocar a coleção e dar suporte às comunicações e intercâmbios na rede; incluindo-se os comput adores e sof tware necessários aos pro tocolos de comunicação;

- A plataforma de colaboração: suporta o trabalho distribuído en tre os vários componentes da rede, incluindo bases de dados específicos, 
Figura 2

Funções do SGC.

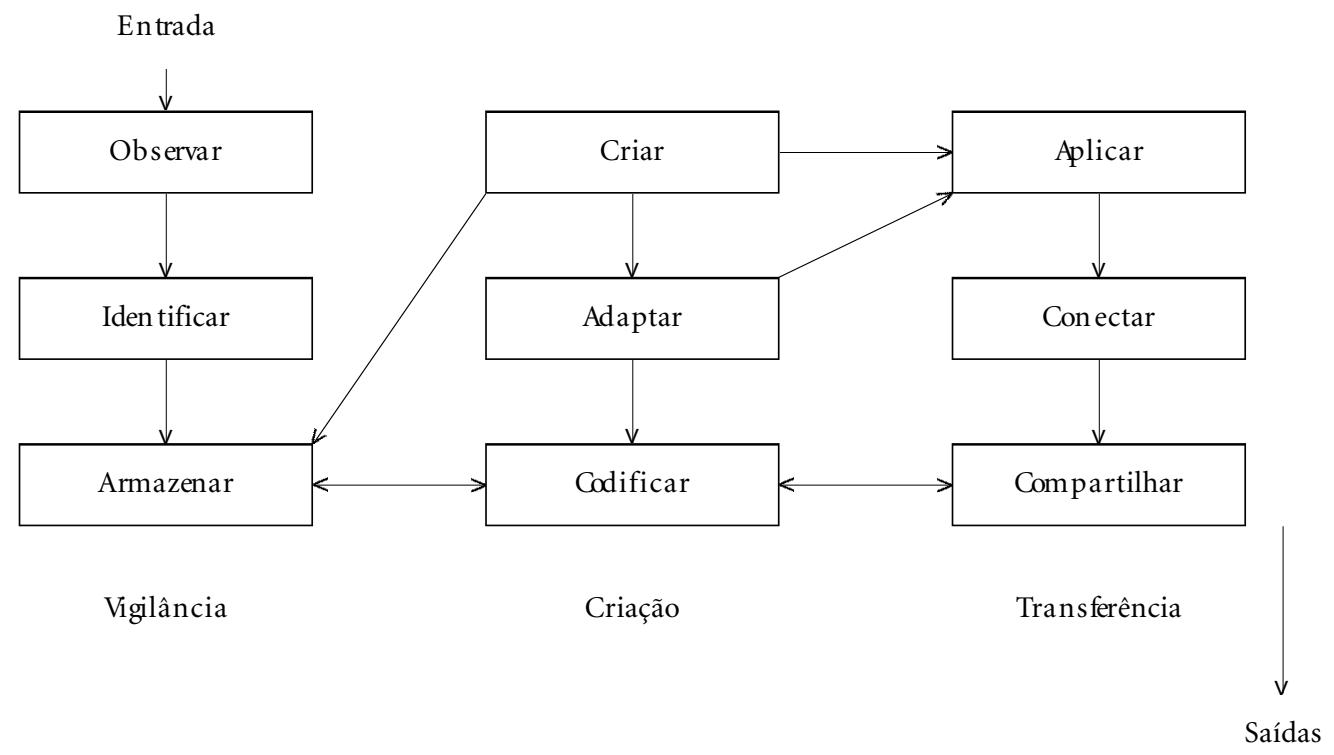

Fon te: Ad a pt ado do estu do de Moore e Bolinches (2001).

grupos de especialistas, construção de espaços virtuais para intercâmbio e cooperação entre as unidades da rede;

- A cultura: con s i derado o fator que decide o êxito ou fracasso dos processos de gestão do conhecimen to. É o resultado da combinação dos valores organizacionais com os pessoais. Aqui é determinantea história prévia da organização, as regras, escritas ou não, e toda a trama de relações que envo lvem o rel acion a men to humano em sociedades.

Para a realização da segunda etapa partiuse da compreensão do processo de con strução da REDEBLH e da iden tificação dos atores que nela desenvolvem atividades, descritos em Maia (2001). Assumiu-se que os componen tes do modelo teóri co escolhido, bem como as suas principais funções formariam o núcleo estrutu ran te do Si s tema de Gestão do Conhecimento da Rede Naci onal de Bancos de Lei te Humano (SGCREDEBLH). Esta opção permitiu realizar um diagnóstico situacional indicativo de prioridades para implantação do sistema e foi efetu ado com base em observações locais reali$z$ adas pelos autores. Em complementação, trabalhou-se com informações adicionais coleta- das em relatórios técnicos, e outras fornecidas por prof is s i onais vinculados a REDEBLH. Desta forma estabeleceu-se a articulação entre a base teórica con ceitual e a aplicação prática.

Na terceira etapa foram utilizados princípios do modelo teórico EO-SECI (Epistemological \& On tological SECI) proposto por López (2002) buscando integração, entre as dimensões epistemológica e ontológica. Obj etivou-se desta forma visualizar os movimen tos do conhecimento. Elegeu-se como campo de observação os resumos de trabalhos científicos publicados nos Anais do IIICongres so Brasilei ro de Bancos de Leite Humano (Fiocruz, 2002). Este even to possibilitou a exposição e o debate de várias modalidades de trabalhos (relatos de experiências, estudos de caso, relatórios de pesquisa, dissertações, teses e outros) de s envo lvidos. Nele, prof is si onais responsáveis pelas mais variadas atividades na Rede exercitaram, de forma interativa, o compartilhamen to do conhecimento que vem sen do gerado tanto na ro tina de sua prática diária como no exercício da ativid adeacadêmica.

A seguir foram utilizadas as funções de obs ervação e identificação, com ponentes do sub- 
sis tema de vigilância, para classificação dos resumos publicados por área temática e tipo de a utoria. Esta análise procurou organizar inicialmen te o caminho necessário à com preensão do s quatro processos de conversão do con hecimento descri tos por Nonaka \& Takeuchi (1995).

Preliminarmente, foram consideradas apenas as modalidades de socialização - conhecimen to tácito a tácito -, que compartilha os conhecimentos tácitos e ocorre através de seminários, jorn adas e outros ti pos de reuniões entre os profissionais; e de ex ternalização - conhecimen to tácito a explícito-, que disponibiliza o conhecimen to tácito ao seu entorno através da análise e inve s ti gação de problemas.

A classificação dos resumos de trabalhos por área tem á tica obj etivou con tri buir para um estudo de base disciplinar, possibilitando associações entre as modalidades de conversão do conhecimen to, ampliando desta forma, a compreensão sobre a dimensão ep is temológica. Ne sta pers pectiva, as áreas temáticas foram identificadas e definidas em função de uma convergência de tendências segundo even tos anteriores, da mesma natureza. Pa ra a análise também foi utilizada a classificação de áreas do conh ecimen to ado tada pelo Con s elho Naci onal de Pe squisa e Desenvo lvim en to Tecnológi co (CNPq) que é a principal agência de fomento a estas a tivid ades no Brasil.

A classificação por perfil de autoria, in dividual e coletiva, obj etivou iniciar o mapeamen to dos distintos níveis ontológi cos a serem considerados para aplicação do modelo EO - SECI proposto por López (2002). Faz-se oportuno de st acar que esta análise não preten deu esgotar a investigação, tanto no que diz respeito aos elementos, como no que tange aos movimentos in erentes à plena compreensão da geração do conhecimento na REDEBLH em suas diferentes dimensões.

\section{Resulta dos e discussão}

No que se refere à definição do modelo teórico de Si s tema de Gestão do Conhecimen tofoi adotado o descritopor Moore \& Bolinches (2001).

Os result ados da etapa de diagnósticosituacional preliminar podem ser ob servados na tabela 1. Foram considerados três dos componen tes de um SGC já descritos, bem como a realid ade atual da REDEBLH. Atribu iu-se a cada um dos atores con cei tos que objetivam indicar pri oridades estratégicas no planejamento da implantação do Sistema (Ma ia , 2004b).

Levando-se em conta os principais subsistemas e as funções específicas de um SGC, já apresentadas na figura 2, bem como a identificação dos atores participantes da REDEBLH descritos em Maia (2004b), pode-se propor um es qu ema preliminar de estrutura funcional para um Sistema de Gestão do Conhecimen to da Rede Nacional de Bancos de Lei te Humano como mostra a tabela 2 .

Os espaços assinalados com $\mathrm{X}$ representam o que é desejável e, portanto, deve ser levado em conta, no planejamen to do SGC, ou seja, que aquele integrante da REDEBLH exerça a corre s pon den te função.

É import a $n$ te de s t acar que este desenho inicial é indicativo e re sulta do en tendimen to que os autores, baseados em suas experiências e prática profissional na REDEBLH, possuem com relação ao tema. Desta forma admite-se que, em razão da dinâmica de implantação do SGC, ou mesmo após sua conclusão, outras fu nções sejam desempenhadas pelos diversos atores com pon en tes do Sis tema.

$\mathrm{Na}$ etapa de simulação foi aplicado o modelo propo s to con forme descritona metodologia, e os resultados são apresentados na tabela 3.

As informações consolidadas na tabela 3 indicam que aproximadamente $90 \%$ dos re su-

Tabela 1

Elemen tos para diagn ó s ti co situ acional na REDEBLH.

\begin{tabular}{lccc}
\hline Atores da Rede & Sede da Rede & Comissões de Al eitamen to & Bancos de Lei te \\
\hline Compon en tes do SGC & & & \\
Coleção & S & A & F \\
Infra-estrutu ra de comunicação & A & A & F \\
Plataforma de colaboração & A & F \\
\hline
\end{tabular}

$\mathrm{S}=$ Suficiente; $\mathrm{A}=$ Necessita ampliação; $\mathrm{F}=$ Insuficien te

Fon te: Maia (2004b) 


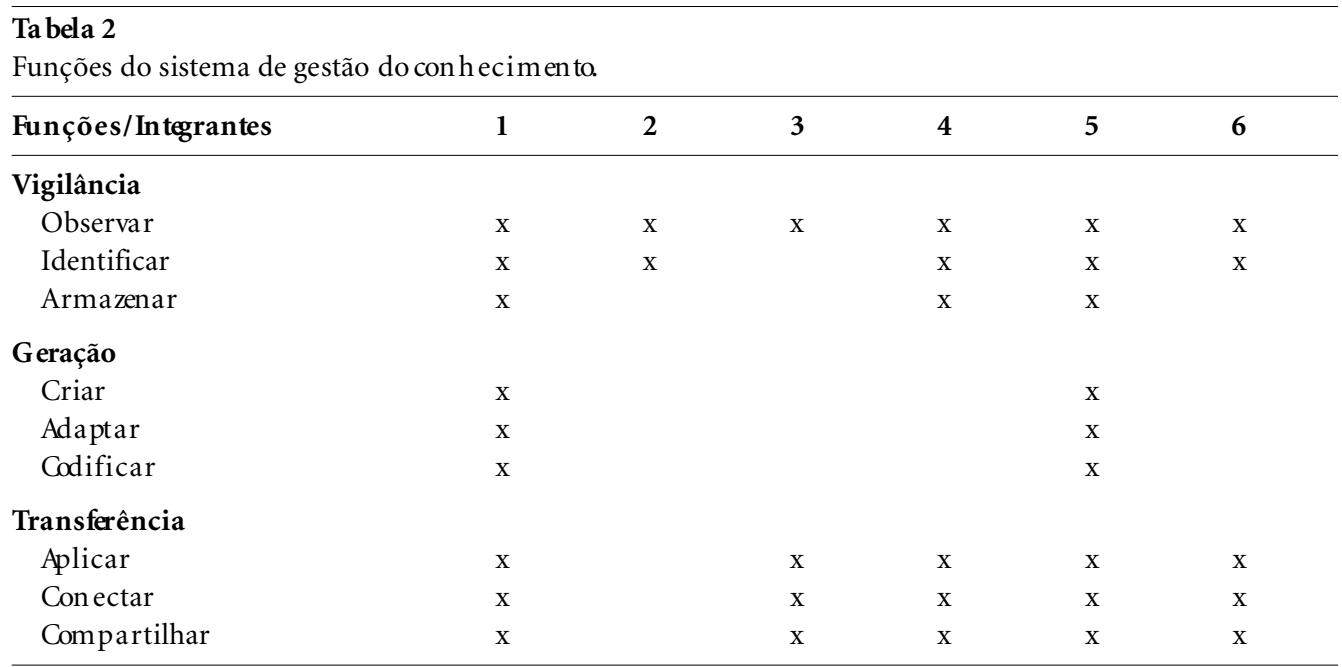

1 - Sede da Rede, Ban cos de Lei te Humano, Comissões de Aleitamento; 2 - Instituições Financeiras;

3 - Gran des Empresas; 4 -As s ociações de Casse; 5 - Gru pos de P\&D; 6 - Organizações não-governamentais. Fon te: Maia (2004b)

\section{Tabela 3}

Distribuição dos resumos apresentados no III Con gresso Brasileiro de Ban cos de Lei te Humano por área temática e tipo de autoria.

\begin{tabular}{lcc}
\hline Área Temática & $\begin{array}{c}\text { Resumos de autoria } \\
\text { individual }\end{array}$ & $\begin{array}{c}\text { Resumos de autoria } \\
\text { em grupo }\end{array}$ \\
\hline 1. Amamentação, Gultura, Gidadania & 18 & 59 \\
2. Assistência à Amamentação & 4 & 51 \\
3. Tecnologia de Alimentos em BLH & 0 & 34 \\
4. Gestão da Qualidade em BLH & 1 & 32 \\
5. In formação/ Comunicação em BLH & 0 & 5 \\
Total & $\mathbf{2 3}$ & $\mathbf{1 8 1}$
\end{tabular}

Fon te: Anais do III Congresso Brasilei ro de Bancos de LeiteHumano, 2002.

mos de trabalhos têm autoria col etiva e provavelmente multiprofissional. As áreas temáticas 1 e 2 , que podem ser con s i deradas como ten do basicamente sua fundamentação nas ciências da saúde e humanas, representam $64 \%$ do total, significando portanto referencial teórico privilegi ado. A área de tecnologia de alimentos, com sua âncora teórica nas ciências biológicas e agrárias, absorve $16 \%$ da produção, com uma tendência predominan temente quantitativa e experimental, exigindo com isso maior tempo para obtenção de resultados e um parque tecnológico mais sofisticado. A área de gestão da qualidade, que por sua vez, en con tra nas ciências sociais aplicadas sua principal fon te teórica, responde por $15 \%$ da produção, demonstra n do uma rel a tiva tendência de consolidação.
A informação e comunicação também edificam sua sustentação teórica nas ciências sociais aplicadas. Contudo, nesta área, abordagens que problemati zem as questões emergentes ainda são bastante recen tes no âmbito da REDEBLH, o que pode explicar o pequ eno número de trabalhos identificados.

A distribuição dos resumos por área temática e região geográfica (tabela 4) evi dencia uma gran de con cen tração (83\%) da produção científica nas regiões Sul e Sudeste, reproduzindo o também desigual quad ro socioecon ô mico que se verifica no país. Este fato corrobora a tendência ob servada no sistema de inovação em saúde no Brasil (Queiroz, 2002). Esta constatação certamente deve ser cuidadosamente considerada para o planejamento do SCG da REDEBLH, so- 
Tabela 4

Distri buição dos re sumos apresentados no III Con gresso Brasilei ro de Ba n cos de Lei te Humano por área temá tica e região geográfica dos autores.

\begin{tabular}{|c|c|c|c|c|c|c|}
\hline Área Temática/Região & Sul & Sudeste & Cen tro-Oeste & Norde s te & Norte & Total \\
\hline 1. Amamentação, Cultura, Gidadania & 11 & 52 & 7 & 6 & 1 & 77 \\
\hline 2. Assistência à Amamentação & 6 & 40 & 0 & 8 & 1 & 55 \\
\hline 3. Tecnologia de Alimentos em BLH & 11 & 20 & 0 & 3 & 0 & 34 \\
\hline 4. Gestão da Qualidade em BLH & 5 & 21 & 2 & 4 & 1 & 33 \\
\hline 5. In formação/Comunicação em BLH & 0 & 5 & 0 & 0 & 0 & 5 \\
\hline Total & 33 & 138 & 9 & 21 & 3 & 204 \\
\hline
\end{tabular}

Fon te: Anais do III Congresso Brasileiro de Bancos de LeiteHumano, 2002.

bretu do em sua função de transferência. Notase que $91 \%$ dos trabalhos da área de tecnologia de alimen tos foram desenvolvi dos nestas regiões. Apenas a região Su des te vem produzindo trabalhos em informação e comunicação.

\section{Considerações finais}

Os resultados evidenciam que é possível e necessá rio implem entar um Sistema de Gestão do Conhecimen to para a REDEBLH. A essência deste sistema é a produção e apropriação do conhecimen to socialmente distribuído. Es te fundamento deve ser garantido pelo trabalho em rede, de modo a articular indivíduos, grupos e instituições que participam da REDEBLH. Foram apon $t$ ados os elementos que pos s ibilitam a es truturação do Sistema. de Gestão do Conhecimento para REDEBLH, cuja implementação deverá con tri buir para a identificação e análise da geração, distribuição e apropriação de s te conhecimen to. Esta op ção, do pon to de vista estratégico, sedimenta o reconhecimento de que a

\section{Colaboradores}

A con cepção original do artigo bem como uma primeira versão foram elaboradas por PR S Maia. Todos os autore s partici i a ram nas revisões e na redação final do artigo.

\section{Agradecimentos}

Os autores agradecem ao Sr. Jua rez Reis, que cedeu espaço e infra-estrutura propícios à realização de s te estu do. matéria-prima que opera a articulação e integração da REDEBLH é o conhecimento.

O panorama descritivo, que foi possível delimitar com a opção metodológica escolhida, indica que o estu do do con hecim en to com partilhado na REDEBLH, além de sua importância como elemen to de integração ao próprio sistema de inovação em saúde do Brasil, é um caminho inve s ti ga tivo importan te para a compreensão do seu processo de conversão e do movimento entre seus níveis. Abre também, a oportunidade para implementação de análise sistemática, reforçando a importância de um Sis tema de Gestão do Con hecimento, que facilite o acesso à inovação. É ainda sustentável afirmar que, em operação, o SGCREDEBLH poderá auxiliar na superação da forte con centração regional da produção de conhecimento a qui con st a tada preliminarmente.

O modelo EO-SECI mostrou-se adequado para aplicação na REDEBLH. Sua utilização, com certeza irá contribuir, de forma efetiva, para estu dos futuros sobre a natu reza e o compartilhamento do conhecimento.

\section{Referências bibli ográficas}

Allix NM 2003. Epistemology and knowled ge management concepts and practices. Journal of Knowledge Management Practice. Volume 4. Disponível em $<$ http://www.tlainc.com/artid49.htm >. Acesso em 1o de junho de 2003.

Almeida JAG, Maia PRS \& Novak FR 2004. Os bancos de leite humano como suporte para a redução da mortalidade infantil - a experiência brasileira. Anais do 2o Congresso Uruguayo de Lactancia Ma terna, 2004 Set; Montevideo, Uruguay. Ed. Sociedad Uruguaya de Pediatria.

Almeida JAG 2001. Breastfeeding: a nature-culture hybrid. Fiocruz, Rio de Janei ro. 
Almeida JAG 1998. Amamentação: repensando o pa radig$m a$. Tese de doutorado. In sti tuto Fern a n desFiguei ra, Fiocruz, Rio de Ja n ei ro.

Campos IM 1997. Gência e tecnologia pa ra a construção da soci edade da info rmação no Bra sil. CNPq, Brasília.

Castells M 2001. A sociedade em rede. Ed. Paz e Terra, Rio de Jan ei ro.

Fiocruz 2002. Pe squisa e desenvolvimento tecnológico em bancos de leite humano. Anais do III Congresso Brasilei ro de Bancos de LeiteHumano. Fiocruz, Rio de Jan eiro.

Giddens A 2000. Mundo em descontrole - o que a globalização está fazendo de nós. Ed. Record, Rio de Ja nei ro.

Kane HCM 2003. Ref raming the knowl ed ge debate, with a little help from the Greeks. Sch ool of In formati on \& Communication Technologies, Un iversity of Paisley, Scotland. Vol. 1, Is sue 1 - Septem ber.

La s tres HMM \& Albagli S 1999. Informação e globalização na era do conhecimento. Ed. Campus, Rio de Ja neiro.

Lastres HMM 2000. Ciência e tecnologia na era do conhecimento: um óbvio papel estratégico. Rev. Pa rcerias Es tratégicas (9):14-21.

Ló pez PV 200 1. La sociedad de la información en América Latina y el Caribe: TICs y un nu evo marco institucional. [CD-Rom]. In Memoria do 9o Seminario Latino-Iberoamericano de Gestión Tecnológica Innovación en la Economia Del Co no cimien to, 2001, outubro; Ed. do Insti tuto Tecnológico da CostaRica, San Jose, Costa Rica.

López JEN, Castro GM, Saez PL \& Muiña FEG 2002. Un modelo integrado de creación y transformación de conocimien to. [CD - ROM] In Memoria do XXII Si mpósio de Gestão da Inovação Tecnológica, 2002, 6 a 8 de novembro, Salvador. Ed. do Núcleo de Política e Gestão Tecnológica, Universidade de São Paulo.

Maia PRS 2004a. Geração, difusão e apropriação do conhecimento na Rede Nacional de Bancos de Leite Hu mano. Tese de do utorado. Instituto Fernan des Figueira, Fiocruz, Rio de Ja n ei ro.

Maia PRS, Novak FR \& Al m eida JAG 2004b. Bases con ceituais da gestão do con hecimento na Rede Nacional de Ba n cos de Leite Humano. Rev Adm Pública 38(2):287306.

Maia PRS 2002. In ovação e gestão do conhecimento na Rede Nacional de Ba n cos de Leite. [CD-ROM] In Memória do XXII Simpósio de Gestão da Inovação Tecnológica, 2002, 6 a 8 de novembro, Salvador. Ed. do Núcleo de Política e Gestão Tecnológica, Un ivers i$\mathrm{d}$ ade de São Paulo.

Maia PRS 2001. Metodologia para avaliação da aplic ação do modelo de redes de inovação na saúde pública um estudo de caso. [CD-ROM] In Memoria do 9o Seminário Latino-Iberoamericano de Gestión Tecnológica In novación en la Economia Del Conocimien to, o utubro; Ed. do In stituto Tecnológico da CostaRica, San Jose, Costa Rica.

Mansell R 1998. Knowled ge societies: information tech nology forsustainable devel opment. Ed. Ox ford Un iversity Press; New York. Disponível em <http://www. sussex.ac.uk/spru/ink/knowl ed ge.html $>$. Acesso em 30 de março de 2005.

Ma rcon des D 2002. Iniciação à história da filosofia: dos pré-socráticos a Wittgenstein. Jor ge Zahar Editor, Rio de Jan ei ro.

Merino JCA, Macedo C 2002. Tra n s ferencia de conocimien to y redes de innovación. [CD-ROM] In Memoria do XXII Simpósio de Gestão da Inovação Tecnológica, 2002, 6 a 8 de novembro, Sa lvador. Ed. do Núcleo de Política e Gestão Tecnológica, Universi dade de São Paulo.

Min ayo MCS 2002. Entre vôos de águia e passos de elefante: caminhos da investigação na atualidade, pp. 17-27. In MCS Min ayo \& SF Deslandes (orgs.). Caminhos do pensamento: epistemologia e método. Fiocruz, Rio de Ja n ei ro.

Moore CES \& Bolinches SB 2001. El desarrollo de un sis tema deges tión del con ocimien to para los insti tutos tecnológicos. [CD-ROM] In Memoria do 9o Seminario Latino - Iberoa mericano de Gestión Tecnológica Innovación en la Economia Del Conocimiento, o utu bro. Ed. do Instituto Tecnológico da Costa Rica, San Jose, Costa Ric a.

Nahapiet J \& Ghoshal S 1998. Social capital, in tellectual capital and theorganizational advantage. Academy of Management Review 23(2):242-266.

Nelson R \& Winter SG 1982. An evolutionary theory of e conomic change. Belknap Press Cambridge.

Nonaka I, Toyama R \& Konno N 2001. SECI, Ba and Le adership: A unified model of dynamic knowl ed ge creation, Part one. In I Nonaka \& DJ Teece (orgs.). Managing industrial knowled ge: creation, transfer and utilization. Sa ge Publications, Lon don.

Nonaka I \& Takeu chi H 1995. The knowledge creating company: how Japanese co mpanies crea te the dynamics of innovation. Ox ford Un iversity Press, New York.

Nonaka I 1994. A dynamic theory of or ga nizational kn owled ge crea tion. Organization Science 5(1):14-37.

Pell egrini A 2000. Cien cia en pro de la salud. Opas, Washington.

Polanyi M 1966. The tacit dimension. Doubleday, New York. Qu ei roz SRR, Bon acelli MBM, Mello DL \& Jolo FS 2002. O CNPq e o sistema de inovação em saúde no Brasil: uma análise a partir dos gru pos de pe squisa do setor saú de [CD-ROM]. In Memoria do XXII Simpósio de Gestão da Inovação Tecnológica, 2002, 6 a 8 de novembro, Sa lvador. Ed. do Núcl eo de Política e Gestão Tecnológica, Un ivers i d ade de São Paulo.

Salazar AAP 2001. Modelo de implantación degestión del conocimiento y tecnologías de información para la generación de ventajas competitivas. Dissertação de mestrado. Departamento de In formática, Un iversi$\mathrm{d}$ ad Técnica Federico Santa Maria, Valparaíso.

Sanchez R 2001. Knowledge management and organizational co mpeten ce. Ox ford Un ivers i ty Press, New York.

Spender JC 1996. Making knowledge: the basis of a dynamic theory of the firm. Strategic Management Journal 17:45-62.

Takahash T 2000. Soci edade da informação no Brasil : livro verde. Mnistério da Ciência e Tecnologia, Brasília.

Valentim MLP et al. 2003. O processo de inteligência com petitiva em organizações. Revista de Ciência da Info rmação 4(3), jun/03. Dis ponível em $<\mathrm{http}: / / \mathrm{www}$. dgzero.org/jun03/Art_03.htm>. Acesso em 4 de abril de 2005.

Virilio P 1996. A arte do motor. Ed. Estação Liberdade, São Paulo.

Zorrilla H 1997. La gerencia del conocimiento y lagestión tecnológica. Dissertação de mestrado. Un iversid adde los Andes, Bogotá.

Artigo apresen t ado em 23/07/2004

Aprovado em 3/03/2005

Versão final apresen tada em 19/04/2005 